\title{
Treatment Options in Bipolar Disorder: Lessons from Population-Based Registers with Focus on Lithium
}

Lars Vedel Kessing, MD, DMSC ${ }^{1,2, *}$

\author{
Address \\ *,1Psychiatric Center Copenhagen Department 0, 6233, Rigshospitalet. Blegdamsvej \\ 9, 2100, Copenhagen, Denmark \\ Email: lars.vedel.kessing@regionh.dk \\ ${ }^{2}$ Faculty of Health and Medical Sciences, University of Copenhagen, Copenhagen, \\ Denmark
}

Published online: 15 July 2015

(C) Springer International Publishing AG 2015

This article is part of the Topical Collection on Mood Disorders

Keywords Bipolar disorder - Lithium - Maintenance $\cdot$ Naturalistic $\cdot$ Population based

\section{Opinion statement}

Population-based register studies have the advantage of large sample sizes and low dropout rates during long-term follow-up. Recent findings from population-based register studies on the efficacy of lithium have supplemented findings from randomized trials and generalized these to all patients with bipolar disorder. Findings from population-based register studies show that (1) half of patients with bipolar disorder continue to take lithium for longer than 6 months, (2) early start of lithium maintenance treatment following the first single manic episode improves the long-term outcome, (3) maintenance treatment with lithium seems to be in general superior to treatment with valproate and lamotrigine, (4) lithium may reduce the risk of suicide and dementia, and (5) lithiuminduced end-stage renal disease is rare with modern lithium treatment within recommended serum levels. Based on the accumulated evidence, lithium should be used as firstline maintenance treatment for bipolar disorder and with intensified group-based psychoeducation following onset of first manic episode/bipolar disorder to improve long-term adherence to lithium, especially among younger patients. 


\section{Why evidence from randomized controlled trials (RCTs) in bipolar disorder should be combined with evidence from observational effectiveness studies}

Within psychiatry, as in the rest of medicine, RCT is the gold standard for obtaining information on how treatment affects health [1]. The validity of RCT results depends on internal validity, i.e., the certainty that the study findings are true for the study population and setting, as well as on external validity, i.e., the generalizability of the findings to other populations and settings [1]. Achieving results from RCTs with high internal and external validity is a major challenge within psychiatry due to the nature of psychiatric illnesses. Specifically, conducting long-term maintenance RCTs in bipolar disorder is extraordinary challenging due to the unpredictable pattern and course of illness with frequent changes in clinical states and periods with remission and recovery compared to major depressive disorders and schizophrenia, which tend to remain stable over months or years [2]. In addition to the complex and labile symptomatic presentations, a tendency for patients to deny illness and reject treatment and diagnostic heterogeneity severely complicate the design and conduct of experimental treatment trials in bipolar disorder as emphasized by Baldessarini [2]. Further, the interpretation and generalization of results from longterm maintenance RCTs in bipolar disorder are severely hampered by the highly selected populations included in such trials [2]. Strict inclusion and exclusion criteria are employed in RCTs to ensure that the randomised treatment is the only major difference between patient groups in order to infer causality between intervention and outcome measures [3]. In this way, the majority of real life patients with bipolar disorder are excluded due to a number of factors including (1) comorbidity with alcohol, cannabis or substance abuse, and panic or obsessivecompulsive disorder; (2) severity of affective episodes including psychotic features; (3) unstable course of illness and rapid cycling states; (4) high risk of suicide; and (5) poor insight with denial or minimisation of illness and poor cooperation with clinical interventions due to impulsivity and impaired judgment $[2,3]$ as well as poor adherence to medication during the run in phase of the RCT. Among patients with major depressive disorder in routine clinical practice between $14[4,5]$ and $34 \%[6]$ meet usual eligibility requirements for an antidepressant efficacy trial. Similar data are not available in relation to bipolar disorder but it is estimated that as few as one in ten of patients with bipolar disorder presenting as potential research subjects will be recruited into a long-term trial involving a placebo condition [2]. RCT designs with an active comparator [7] are likely to include a slightly higher, approaching $20 \%$, proportion bipolar of population treated in routine clinical care [8].

At least six additional and important factors further limit the generalizability of findings from maintenance RCTs in bipolar disorder: (1) the duration of all currently available RCTs is less than 2 years in contrast to the life long course of bipolar disorder; (2) most RCTs are conducted within tertiary university settings treating patients with the more complicated and/or treatment resistant disorders; (3) maintenance RCTs in bipolar disorder, with very few exceptions such as the Balance trial [9], employ enriched designs favoring the drug of interest based on its prior effect or tolerability during an affective episode [10]; (4) the vast majority of maintenance RCTs are industry initiated resulting in few head to head comparing RCTs [7]; (5) the effectiveness of any medication estimated from highly controlled randomized data selectively submitted to regulatory agencies [11] is often substantially less than its real-world efficacy; (6) finally, realistically, only a small number of large scale RCTs will ever be conducted compared to the number of important questions.

In conclusion, evidence from RCTs has to be combined with evidence from observational studies of patients with bipolar disorder treated in real-world clinical practice, i.e., employing a naturalistic design. In a recent statement, the US National Institute of Health committed itself to comparative effectiveness research and large scale observational studies aiming to generate viable hypotheses, confirm results of randomized trials in understudied patient subsets, learn about rare events, and gain insight into processes of care delivery [12]. Unlike RCTs, observational effectiveness studies are subject to selection and confounding factors due to initial differences in the compared groups while both types of studies share problems with attrition and dropouts during follow-up [1]. However, observational effectiveness studies using population-based 
registers, particularly when using nationwide data, offer a number of possibilities to investigate the generalizability of findings from RCTs in other populations and settings. The major advantage is that such population based registers are collecting patient data on an individual level as part of daily clinical routine on all citizens in the country as long as they do not emigrate out of the country resulting in full attrition and very low dropout rates during followup [13-16]. In this way, the best of such nation-wide registers are fully inclusive and exclusive, i.e., they include $100 \%$ of the population and no one else. Additionally, population-based registers may offer longitudinal data over many decades, which is highly difficult and costly to achieve in other ways. Finally, such data are collected independently of patients and researchers eliminating recall and observational bias.

The aim of the present review was to outline examples of how evidence from maintenance RCTs and population-based registers can be combined to guide clinicians in their treatment choices and procedures in bipolar disorder. In addition, the paper presents examples of how an epidemiological analytical approach to population-based registers can help to elucidate hypotheses on the effect of drugs on long-term outcomes such as suicide and dementia. The review focuses on lithium maintenance long-term treatment in bipolar disorder.

Although lithium has been used as a mood stabilizing treatment of bipolar disorder for more than 60 years, it has been a controversial drug since its introduction in 1949 [17-19]. The evidence from RCTs for a mood stabilizing effect of lithium has increased further during recent years $[9,10,20]$. Similarly, a number of population-based register studies have contributed with important new information including data on the question of generalizability. The aim of the present review was to summarize findings from population-based register studies on the use of lithium in clinical practice.

\section{Adherence to Lithium in Clinical Practice}

Adherence to lithium has been investigated in a number of clinical samples including up to 400 patients [21] mainly from specialized mood disorder clinics or lithium clinics at academic medical centers [21-26]. Therefore, it is likely that there is a selection bias toward patients with the most severe illness. A 6-year pharmacoepidemiological study in the metropolitan area of Portland, USA found a substantially low rate of adherence to lithium among 1594 patients with a median length of adherence of 72 days $(95 \% \mathrm{CI}=66-80)$ [27]. As data were obtained from a private prepaid health maintenance organization (HMO), it is possible that these findings may not generalize to all socioeconomic groups [27].

In a Danish nation-wide population-based register study, we identified more than 14,000 individuals who were prescribed lithium among the 5.3 million persons living in Denmark between 1995 and 2000; in this sample, median time to discontinuation of lithium was 181.0 days ( $95 \% \mathrm{CI}=135.7$ 181.0) [28]. The advantage of this study is that it minimized ascertainment or help-seeking biases as it included all individuals within the country. Moreover, the Danish population is ethnically and socially homogeneous. Psychiatric treatment is available free of charge in Denmark, and lithium is cheap with $75 \%$ of the cost being refunded from the state. In this way, it is likely that the findings can be extrapolated to patients treated with lithium in general. No other study on adherence to lithium has been published for an entire population in a country. We further found that adherence to lithium was significantly poorer for younger (18-39 years) and for older patients ( $\geq 60$ years) compared to middleaged patients [28]. In fact, we subsequently confirmed in a RCT of early intervention in bipolar disorder that adults younger than 25 years have a poorer prognosis 
and adherence to medication when treated in standard care [29]. Taken together, these results highlight the need for increased focus on long-term adherence to lithium with intensified psychological support especially among younger patients. Interestingly, adherence to medication and prognosis can be improved among younger adults with bipolar disorder. In the RCT mentioned above, young adults randomised to treatment in a specialized outpatient mood disorder clinic combining optimized pharmacological treatment and group psychoeducation had substantially improved prognosis and adherence to medication compared to those randomised to standard care (67 \% less risk of rehospitalisation during follow-up, [29], see also [30]).

\section{Response to Lithium in Clinical Practice}

Not all patients experience a mood stabilizing effect from lithium. Some patients develop side effects or have a poor therapeutic response whereas others tolerate lithium well and experience excellent response with a nearly complete prevention of episodes. Studies on response to lithium and on predictors of response to lithium have been influenced by a number of shortcomings [31]: (1) small sample sizes (from 7 to 360 patients [32]), (2) relatively short follow-up periods (i.e., less than 2 years with a few exceptions with up to 5 or 7 years and one study covering a span of up to 20 years [31], (3) selection bias as most samples of patients are recruited form tertiary university specialized centers [31], and (4) high drop-out rates during follow-up.

We presented data on excellent response to lithium monotherapy (i.e., "cure" from future affective episodes), from a population-based and nation-wide register linkage study [33•]. Excellent lithium responders were defined as patients who following a 6-month stabilization start-up period of lithium treatment, continued with lithium as monotherapy without getting hospitalized. Among a total of 3327 patients with bipolar disorder included in the study, the prevalence of patients with an excellent response to lithium was $6.4 \%$ during a median follow-up period of 6.6 years (quartiles; 4.0-9.3 years). This prevalence is lower than the estimated $30 \%$ response rate found in many clinical samples [34], but few of the long-term studies have reported the actual response rates among all patients initiated on lithium (intension to treat analyses). One study that did so examined 402 patients who started lithium in a mood disorder clinic and were followup for up to 5 years. The study reported a response rate of $19.4 \%$, i.e., $19.4 \%$ of the 402 patients had no affective episodes or were taking other psychotropic drugs in addition to lithium [21]. Most likely, the results from the Danish study are more representative of patients treated with lithium in general. Thus, the study included all patients with a diagnosis of bipolar disorder within psychiatric hospital health care settings who initiated lithium treatment during a study period for up to 12 years for all outpatient settings, i.e., within psychiatric hospital outpatients, community psychiatric centers, private specialist practice, and general practice. It was concluded that the prevalence of excellent response to lithium monotherapy is low during long-term treatment of 
more than 5 years. Further, we found that patients with excellent lithium response were characterized by few prior psychiatric hospitalizations, a manic index episode prior to lithium and reduced comorbidity in accordance with other studies [34].

\section{When should Maintenance Treatment with Lithium Start?}

The nature of bipolar disorder seems to imply the presence of an active process of neuroprogression that is considered to be at least partly mediated by inflammation, oxidative stress, apoptosis, and changes in neurogenesis [35]. A wealth of data suggests that lithium may facilitate neural plasticity [36] suggesting advantages in early intervention with the drug. However, it is unclear when to start prophylaxis with lithium. Not all patients will suffer from an additional episode and the number needed to treat (NNT) will by higher early in the course of illness as the risk is lower [10]. Further, it is often claimed that the acceptance of prophylactic lithium treatment may be low in the early stages [10]. Accordingly, in standard mental health care, adherence to lithium is poorer for younger individuals $[28,29]$. However, new randomised data suggest that this low adherence may be turned around to a very high adherence when younger patients aged 18-25 years with bipolar disorder are offered combined optimised pharmacological treatment and group-based psychoeducation in a specialised mood disorder clinic further improving the long-term outcome [29].

No RCT has investigated the effect of lithium intervention early versus late in bipolar disorder. Recommendations of when preventive treatment should be initiated differ substantially among guidelines although most guidelines do not specify when long-term prophylactic treatment should be initiated [10]. We recently conducted a population-based register-based study to try to elucidate the issue comparing response rates among patients with bipolar disorder starting lithium early versus late $[37 \bullet \bullet]$. As in our prior study [33•], lithium responders were defined as patients who following 6 months stabilization start-up period of lithium treatment, continued lithium as monotherapy without getting hospitalized. Early versus late intervention was defined in two different ways: (1) start of lithium following first contact and (2) start of lithium following a diagnosis of a single manic/mixed episode. The study included all patients with a diagnosis of bipolar disorder in psychiatric hospital settings who were prescribed lithium during a period from 1995 to 2012 in Denmark $(N=4714)$. Patients who started lithium early had significantly decreased rates of non-response to lithium compared to the rate for patients starting lithium later (adjusted analyses-first versus later contact, $p<0.0001 ; \mathrm{HR}=0.87,95 \% \mathrm{CI}=0.76-0.91$; single manic $/$ mixed episode versus bipolar disorder $p<0.0001$; $\mathrm{HR}=0.75,95 \% \mathrm{CI}=0.67$ $0.84)$. The findings seem to indicate that lithium may counteract neuroprogression when started early compared to when lithium is started at a later stage. We concluded that lithium initiation following the first psychiatric contact or following diagnosis of a single manic/ 
mixed episode is associated with increased probability of lithium response. This finding clearly needs to be replicated in a future RCT or in additional observational studies.

\section{Lithium versus Valproate in Maintenance Treatment for Bipolar Disorder}

Valproate is often used as mood stabilizers in bipolar disorder. For example, in the USA, valproate is the most used mood stabilizer prescribed as monotherapy followed by lithium [38]. The evidence for the effectiveness of valproate remains somewhat sparse. Two maintenance RCTs on valproate have been published. In the only placebo-controlled monotherapy maintenance study, neither valproate nor lithium showed clear prophylactic effects compared to placebo during 1-year follow-up [39]. In the 2-year long BALANCE study, lithium was more effective than valproate [9] in a mixed population, some of whom were also receiving other long-term medicines. We compared the effect of valproate versus lithium for the treatment of bipolar disorder in clinical practice in a population-based register study [40•]. The two outcomes considered were the rates of switch to, or addition of, another psychotropic and psychiatric hospitalization. Hospitalization is an important hard outcome almost always indicating significant treatment failure. When patients start with valproate or lithium as monotherapy, the addition of another drug indicates that the initial drug was not sufficient to improve affective symptoms or maintain remission, or in the case of switch, there was inadequate response or intolerable side effects. The rate of switch/add on to the comparator drug (from lithium to valproate or the reverse), antidepressants, antipsychotics, or other anticonvulsants, was increased for valproate compared with lithium ( $\mathrm{HR}=1.86$ (95\% CI=1.59-2.16)). Similarly, the rate of psychiatric hospitalizations was increased for valproate versus lithium $(\mathrm{HR}=1.33(95 \% \mathrm{CI}=1.18-1.48))$ regardless of the type of episode leading to hospitalisation (depressive or manic/mixed). We minimized the potential impact of prior treatment by selecting only patients receiving lithium or valproate for the first time. We adjusted the analyses for a large number of variables including gender; age group in 5-year intervals; years since diagnosis; redemption of prescriptions of antidepressants, antipsychotics, or anticonvulsants other than valproate prior to initiating treatment with lithium or valproate; and previous number of psychiatric hospitalizations. We cannot exclude potential confounders associated with treatment allocation to lithium or valproate since this was not random. Nevertheless, we concluded that in daily clinical practice, treatment with lithium seems to be in general superior to treatment with valproate.

\section{Lithium versus Lamotrigine in Maintenance Treatment for Bipolar Disorder}

Two 18-month maintenance RCTs comparing lamotrigine, lithium, and placebo have found an advantage for lamotrigine for the prevention of depressive recurrence while lithium was superior in the prevention of manic recurrence 


\section{Does Lithium Protect against Suicide?}

$[41,42]$. Both studies used partly enriched designs favoring lamotrigine as only patients who tolerated lamotrigine during the open-label phase were randomized. A Danish study without any enrichment found no differences in maintenance effectiveness between lamotrigine and lithium regardless of whether the outcome episode was manic or depressive [43]. We used a similar design, outcome measures, and statistical analyses as in the study comparing lithium to valproate mentioned above [40•] in order to compare rates of switch to, or addition of, another psychotropic, and rates of psychiatric hospitalization for patients treated with lamotrigine or lithium in clinical practice [44•]. The overall rate of switch to or add on of another psychotropic (the opposite drug of interest (lithium or lamotrigine), antidepressants, antipsychotics, or other anticonvulsants than lamotrigine) was increased for lamotrigine compared with lithium $(\mathrm{HR}=2.60,95 \% \mathrm{CI}=2.23-3.04)$ regardless of whether the index episode was depressive, manic, mixed, or remission. Additionally, the overall rate of psychiatric hospitalization was increased for lamotrigine compared with lithium (HR=1.45, $95 \% \mathrm{CI}=1.28-1.65)$.

In a meta-analysis of 48 RCTs including 6674 participants, lithium was found to be more effective than placebo in reducing the number of suicides (odds ratio $0.13,95 \%$ confidence interval 0.03 to 0.66$)$ and deaths from any cause (0.38, 0.15 , to 0.95$)$ [45].

However, such RCTs are not representative of patients with bipolar disorder in general, as patients who are at high risk of suicide are not included in these trials. Nevertheless, observational studies have confirmed that treatment with lithium may be associated with reduced risk of suicide in bipolar disorder also at a nationwide population-based level $[46,47]$. Further, these studies found a dose response association; patients who redeemed lithium prescriptions had higher rates of suicide than persons who did not redeem lithium prescriptions. Redeeming lithium prescriptions at least twice was associated with a 0.44 (95\% $\mathrm{CI}=0.28-0.70)$ reduced rate of suicide, compared to once only, and there was a further decrease in the rate of suicide with increasing number of redeemed lithium prescriptions [46]. Lithium may exert its antisuicidal effects in bipolar disorder by reducing relapses. Additional mechanisms should also be considered as there is some evidence that lithium decreases aggression and possibly impulsivity, which might be another mechanism mediating the antisuicidal effect [45].

\section{Is Bipolar Disorder Associated with Dementia?}

Data suggest that patients with bipolar disorder have twofold increased risk of developing dementia. Out of four prior studies [48-51], the three populationbased studies [49-51] found a positive association between bipolar disorder and dementia and pooling all these data in a meta-analysis confirmed the association $[52 \bullet \bullet]$. One study specifically found that the risk of developing dementia increased with each new affective episode [53]. More recently, a fifth population-based study further confirmed the association between bipolar disorder and dementia even after adjustment for important covariates including 
cerebrovascular disease, diabetes mellitus, hypertension, head injury, chronic pulmonary disease, alcohol-related disorder, substance-related disorder, and number of inpatient and outpatient visits [54].

\section{Does Lithium Protect against Dementia?}

Data is emerging suggesting that lithium may reduce the risk of dementia which may be related to the proposed role of the drug in neuroprotection (see, e.g., [55]). Two Danish register-based population studies found that lithium may ameliorate the risk of subsequent dementia and Alzheimer's disease at the level of the general population $[56 \bullet, 57]$. There is also a randomized trial suggesting that lithium may prevent the development of Alzheimer's disease in patients with mild cognitive impairment (MCI) [58]. On the other hand, a 39-week observational study [59] and a 10-week RCT [60] found no effect of lithium in adults with mild to moderate Alzheimer's disease. These findings indicate that the neuroprotective effect of lithium may be either limited to the very earlystage of Alzheimer's disease or that it requires longer-term exposure. Supportive of the latter hypothesis, a small RCT showed that lithium at extremely low doses (300 $\mu$ g once daily) for 15 months prevented cognitive decline in patients with Alzheimer's disease [61].

\section{Lithium and Renal Failure}

In the hitherto largest and only controlled study, 30 patients with end-stage renal disease were identified among 3836 patients treated with lithium, representing an eightfold increase in prevalence compared with the general population [62]. Unfortunately, this result could have been confounded by recall bias, as data on lithium was partly based on recall from patients, and by the clinical practice of aiming for high serum lithium levels (between 0.8 and $1.2 \mathrm{mmol} / \mathrm{l}$ ) as was recommended in the 1960s, 1970s, and 1980s as none of the 30 patients with end-stage renal disease started lithium treatment later than 1980. The authors concluded that modern lithium treatment guidelines that recommend much lower serum levels may have eliminated the risk of lithiuminduced end-stage renal disease and therefore advocated the continued use of lithium as a safe drug for the long-term treatment of mood disorders [63]. This conclusion is in accordance with the findings in a decision analysis model based on a systematic literature review investigating whether either lithium or an anticonvulsant should be used for maintenance treatment for bipolar disorder if the risks of suicide and relapse were traded off against the risk of end-stage renal disease [64]. The study concluded that at the current state of knowledge, lithium initiation and continuation even in the presence of long-term adverse renal effects should be recommended in most cases [64].

\section{Conclusion}

Findings from population-based register studies show that (1) half of the patients with bipolar disorder continue to take lithium for longer than half a year, (2) starting lithium maintenance soon after the first single manic episode 
improves the long-term outcome, (3) in general, maintenance treatment with lithium seems to be superior to treatment with valproate or lamotrigine, (4) lithium may reduce the risk of suicide and dementia, and (5) lithium-induced end-stage renal disease is rare with modern lithium treatment within recommended serum levels.

In this way, findings from population-based register studies have substantiated and supplemented findings from randomized trials and demonstrated their relevance to patients with bipolar disorder in general. Based on the accumulated evidence, following onset of first manic episode/bipolar disorder, lithium should be used as first-line maintenance treatment for bipolar disorder coupled with intensified group-based psychoeducation to improve long-term adherence to lithium, especially among younger patients.

\section{Compliance with Ethics Guidelines}

\section{Conflict of Interest}

Unrelated to this article, Lars Vedel Kessing has within the preceding 3 years been a consultant for Lundbeck and AstraZenica and has received payment for lectures including service on speakers' bureaus. Lars Vedel Kessing declares that he has no conflict of interest.

\section{Human and animal rights and informed consent}

This article does not contain any studies with human or animal subjects performed by any of the authors.

\section{References and Recommended Reading}

Papers of particular interest, published recently, have been highlighted as:

- Of importance

- Of major importance

1. Wells KB. Treatment research at the crossroads: the scientific interface of clinical trials and effectiveness research. Am J Psychiatry. 1999;156(1):5-10.

2. Baldessarini RJ. Treatment research in bipolar disorder: issues and recommendations. CNS Drugs. 2002;16(11):721-9.

3. Reed C, Novick D, Gonzalez-Pinto A, Bertsch J, Haro JM. Observational study designs for bipolar disorder-what can they tell us about treatment in acute mania? Prog Neuropsychopharmacol Biol Psychiatry. 2009;33(4):715-21.

4. Zimmerman M, Mattia JI, Posternak MA. Are subjects in pharmacological treatment trials of depression representative of patients in routine clinical practice? Am J Psychiatry. 2002;159(3):469-73.

5. Keitner GI, Posternak MA, Ryan CE. How many subjects with major depressive disorder meet eligibility requirements of an antidepressant efficacy trial? J Clin Psychiatry. 2003;64(9):1091-3.
6. Partonen T, Sihvo S, Lonnqvist JK. Patients excluded from an antidepressant efficacy trial. J Clin Psychiatry. 1996;57(12):572-5.

7. Reinares M, Vieta E, Colom F, Martinez-Aran A, Torrent $\mathrm{C}$, Comes $\mathrm{M}$, et al. Impact of a psychoeducational family intervention on caregivers of stabilized bipolar patients. Psychother Psychosom. 2004;73(5):312-9.

8. Henry C, Andreassen OA, Barbato A, DemotesMainard J, Goodwin GM, Leboyer M, et al. A network to foster research and promote innovative care. Int J Bipolar Disord. 2013;1:2.

9. Geddes JR, Goodwin GM, Rendell J, Azorin JM, Cipriani A, Ostacher MJ, et al. Lithium plus valproate combination therapy versus monotherapy for relapse prevention in bipolar I disorder (BALANCE): a randomised open-label trial. Lancet. 2010;375(9712):385-95.

10. Grunze H, Vieta E, Goodwin GM, Bowden C, Licht RW, Moller HJ, et al. The World Federation of Societies of Biological Psychiatry (WFSBP) guidelines for the biological treatment of bipolar disorders: update 2012 on 
the long-term treatment of bipolar disorder. World J Biol Psychiatry. 2013;14(3):154-219.

11. Calabrese JR, Kemp DE. Bipolar drug development: are we getting closer to the real world? Am J Psychiatry. 2008;165(10):1234-6.

12. Lauer MS, Collins FS. Using science to improve the nation's health system: NIH's commitment to comparative effectiveness research. JAMA.

2010;303(21):2182-3.

13. Munk-Jorgensen P, Kastrup M, Mortensen PB. The Danish psychiatric register as a tool in epidemiology. Acta Psychiatr Scand Suppl. 1993;370:27-32.

14. Munk-Jorgensen P, Mortensen PB. The Danish Psychiatric Central Register. Dan Med Bull. 1997;44(1):82-4

15. Mors O, Perto GP, Mortensen PB. The Danish Psychiatric Central Research Register. Scand J Public Health. 2011;39(7 Suppl):54-7.

16. Allebeck P. The use of population based registers in psychiatric research. Acta Psychiatr Scand. 2009;120(5):386-91.

17. Cade JF. Lithium salts in the treatment of psychotic excitement. Med J Aust. 1949;2(10):349-52.

18. Malhi GS. The king is dead, long live the king! The restoration of BALANCE. Bipolar Disord.

2010;12(7):681-4.

19. Ghaemi SN. From BALANCE to DSM-5: taking lithium seriously. Bipolar Disord. 2010;12(7):673-7.

20. Coryell W. Maintenance treatment in bipolar disorder: a reassessment of lithium as the first choice. Bipolar Disord. 2009;11 Suppl 2:77-83.

21. Maj M, Pirozzi R, Magliano L, Bartoli L. Long-term outcome of lithium prophylaxis in bipolar disorder: a 5 -year prospective study of 402 patients at a lithium clinic. Am J Psychiatry. 1998;155(1):30-5.

22. Goodwin FK, Jamison KR. Manic-depressive illness. Oxford: Oxford University Press; 1990.

23. Lingam R, Scott J. Treatment non-adherence in affective disorders. Acta Psychiatr Scand. 2002;105(3):164-72.

24. Aagaard J, Vestergaard P. Predictors of outcome in prophylactic lithium treatment: a 2-year prospective study. J Affect Disord. 1990;18(4):259-66.

25. Berghofer A, Kossmann B, Muller-Oerlinghausen B. Course of illness and pattern of recurrences in patients with affective disorders during long-term lithium prophylaxis: a retrospective analysis over 15 years. Acta Psychiatr Scand. 1996;93(5):349-54.

26. Schumann C, Lenz G, Berghofer A, MullerOerlinghausen $\mathrm{B}$. Non-adherence with long-term prophylaxis: a 6-year naturalistic follow-up study of affectively ill patients. Psychiatry Res. 1999;89(3):247-57.

27. Johnson RE, McFarland BH. Lithium use and discontinuation in a health maintenance organization. Am J Psychiatry. 1996;153(8):993-1000.

28. Kessing LV, Sondergard L, Kvist K, Andersen PK. Adherence to lithium in naturalistic settings: results from a nationwide pharmacoepidemiological study. Bipolar Disord. 2007;9(7):730-6.

29. Kessing LV, Hansen HV, Christensen EM, Dam H, Gluud C, Wetterslev J. Do young adults with bipolar disorder benefit from early intervention? J Affect Disord. 2014;152-154:403-8.

30. Kessing LV, Hansen HV, Hvenegaard A, Christensen EM, Dam H, Gluud C, et al. Treatment in a specialised out-patient mood disorder clinic v. standard outpatient treatment in the early course of bipolar disorder: randomised clinical trial. Br J Psychiatry. 2013;202:212-9.

31. Berghofer A, Alda M, Adli M, Baethge C, Bauer M, Bschor $\mathrm{T}$, et al. Long-term effectiveness of lithium in bipolar disorder: a multicenter investigation of patients with typical and atypical features. J Clin Psychiatry. 2008;69(12):1860-8.

32. Kleindienst N, Engel R, Greil W. Which clinical factors predict response to prophylactic lithium? A systematic review for bipolar disorders. Bipolar Disord. 2005;7(5):404-17.

33. Kessing LV, Hellmund G, Andersen PK. Predictors of excellent response to lithium: results from a nationwide register-based study. Int Clin Psychopharmacol. 2011;26(6):323-8.

This study identifies predictors of lithium response in a nationwide population.

34. Rybakowski JK. Factors associated with lithium efficacy in bipolar disorder. Harv Rev Psychiatry. 2014;22(6):353-7.

35. Berk M, Berk L, Dodd S, Cotton S, Macneil C, Daglas R, Conus P, Bechdolf A, Moylan S, Malhi GS: Stage managing bipolar disorder. Bipolar Disord 2013.

36. Gray JD, McEwen BS. Lithium's role in neural plasticity and its implications for mood disorders. Acta Psychiatr Scand. 2013;128(5):347-61.

37.• Kessing LV, Vradi E, Andersen PK. Starting lithium prophylaxis early v. late in bipolar disorder. Br J Psychiatry. 2014;205(3):214-20.

This study is the first to investigate response rates to lithium when started early versus late in bipolar disorder.

38. Baldessarini R, Henk H, Sklar A, Chang J, Leahy L. Psychotropic medications for patients with bipolar disorder in the United States: polytherapy and adherence. Psychiatr Serv. 2008;59(10):1175-83.

39. Bowden CL, Calabrese JR, McElroy SL, Gyulai L, Wassef A, Petty F, et al. A randomized, placebo-controlled 12 month trial of divalproex and lithium in treatment of outpatients with bipolar I disorder. Divalproex Maintenance Study Group. Arch Gen Psychiatry. 2000;57(5):481-9.

40. Kessing LV, Hellmund G, Geddes JR, Goodwin GM, Andersen PK. Valproate v. lithium in the treatment of bipolar disorder in clinical practice: observational nationwide register-based cohort study. Br J Psychiatry. 2011;199:57-63.

41. Bowden CL, Calabrese JR, Sachs G, Yatham LN, Asghar SA, Hompland M, et al. A placebo-controlled 18month trial of lamotrigine and lithium maintenance treatment in recently manic or hypomanic patients with bipolar I disorder. Arch Gen Psychiatry. 2003;60(4):392-400. 
42. Calabrese JR, Bowden CL, Sachs G, Yatham LN, Behnke $\mathrm{K}$, Mehtonen OP, et al. A placebo-controlled 18-month trial of lamotrigine and lithium maintenance treatment in recently depressed patients with bipolar I disorder. J Clin Psychiatry. 2003;64(9):1013-24.

43. Licht RW, Nielsen JN, Gram LF, Vestergaard P, Bendz $\mathrm{H}$. Lamotrigine versus lithium as maintenance treatment in bipolar I disorder: an open, randomized effectiveness study mimicking clinical practice. The 6 th trial of the Danish University Antidepressant Group (DUAG-6). Bipolar Disord. 2010;12(5):483-93.

44. $\quad$ Kessing LV, Hellmund G, Andersen PK. An observational nationwide register based cohort study on lamotrigine versus lithium in bipolar disorder. J Psychopharmacol. 2012;26(5):644-52.

This study compares long-term effects of maintenance treatment with lithium and lamotrigine in clinical practice.

45. Cipriani A, Hawton K, Stockton S, Geddes JR. Lithium in the prevention of suicide in mood disorders: updated systematic review and meta-analysis. BMJ. 2013;346:f3646.

46. Kessing LV, Sondergard L, Kvist K, Andersen PK. Suicide risk in patients treated with lithium. Arch Gen Psychiatry. 2005;62(8):860-6.

47. Sondergard L, Lopez AG, Andersen PK, Kessing LV. Mood-stabilizing pharmacological treatment in bipolar disorders and risk of suicide. Bipolar Disord. 2008;10(1):87-94.

48. Kokmen E, Beard CM, Chandra V, Offord KP, Schoenberg BS, Ballard DJ. Clinical risk factors for Alzheimer's disease: a population-based case-control study. Neurology. 1991;41(9):1393-7.

49. Cooper B, Holmes C. Previous psychiatric history as a risk factor for late-life dementia: a population-based case-control study. Age Ageing. 1998;27(2):181-8.

50. Kessing LV, Olsen EW, Mortensen PB, Andersen PK. Dementia in affective disorder: a case-register study. Acta Psychiatr Scand. 1999;100(3):176-85.

51. Kessing LV, Nilsson FM. Increased risk of developing dementia in patients with major affective disorders compared to patients with other medical illnesses. J Affect Disord. 2003;73(3):261-9.

$52 . \bullet$ da Silva J, Goncalves-Pereira M, Xavier M, MukaetovaLadinska EB. Affective disorders and risk of developing dementia: systematic review. Br J Psychiatry. 2013;202:177-86.

This systematic review and meta-analysis presents pooled data on the association between affective disorders and subsequent dementia.

53. Kessing LV, Andersen PK. Does the risk of developing dementia increase with the number of episodes in patients with depressive disorder and in patients with bipolar disorder? J Neurol Neurosurg Psychiatry. 2004;75(12):1662-6.

54. Wu KY, Chang CM, Liang HY, Wu CS, Chia-Hsuan WE, $\mathrm{Chen} \mathrm{CH}$, et al. Increased risk of developing dementia in patients with bipolar disorder: a nested matched case-control study. Bipolar Disord. 2013;15(7):78794.

55. Hado-Vieira R, Manji HK, Zarate Jr CA. The role of lithium in the treatment of bipolar disorder: convergent evidence for neurotrophic effects as a unifying hypothesis. Bipolar Disord. 2009;11 Suppl 2:92-109.

56. Kessing LV, Sondergard L, Forman JL, Andersen PK. Lithium treatment and risk of dementia. Arch Gen Psychiatry. 2008;65(11):1331-5.

This population based study presents an analytical design aiming to decrease potential confounding by indication in the association between lithium and dementia.

57. Kessing LV, Forman JL, Andersen PK. Does lithium protect against dementia? Bipolar Disord. 2010;12(1):87-94.

58. Forlenza OV, Diniz BS, Radanovic M, Santos FS, Talib LL, Gattaz WF. Disease-modifying properties of longterm lithium treatment for amnestic mild cognitive impairment: randomised controlled trial. Br J Psychiatry. 2011;198:351-6.

59. Macdonald A, Briggs K, Poppe M, Higgins A, Velayudhan L, Lovestone S. A feasibility and tolerability study of lithium in Alzheimer's disease. Int J Geriatr Psychiatry. 2008;23(7):704-11.

60. Hampel H, Ewers M, Burger K, Annas P, Mortberg A, Bogstedt A, et al. Lithium trial in Alzheimer's disease: a randomized, single-blind, placebo-controlled, multicenter 10-week study. J Clin Psychiatry.

2009;70(6):922-31.

61. Nunes MA, Viel TA, Buck HS. Microdose lithium treatment stabilized cognitive impairment in patients with Alzheimer's disease. Curr Alzheimer Res.

2013;10(1):104-7.

62. Aiff H, Attman PO, Aurell M, Bendz H, Schon S, Svedlund J. End-stage renal disease associated with prophylactic lithium treatment. Eur

Neuropsychopharmacol. 2014;24(4):540-4.

63. Aiff H, Attman PO, Aurell M, Bendz H, Schon S, Svedlund J. The impact of modern treatment principles may have eliminated lithium-induced renal failure. J Psychopharmacol. 2014;28(2):151-4.

64. Werneke U, Ott M, Renberg ES, Taylor D, Stegmayr B. A decision analysis of long-term lithium treatment and the risk of renal failure. Acta Psychiatr Scand. 2012;126(3):186-97. 